\title{
Diagnoseverzögerung bei Brustkrebs
}

\author{
Die Erben einer an ihrer Krebserkrankung verstorbenen Patientin \\ forderten Schadensersatz und Schmerzensgeld, da ein bösartiger \\ Brusttumor zu spät erkannt worden sei.
}

ie Patientin hatte sich am 16.7.2012 mit Schmerzen in der rechten Brust vorgestellt. Die Tastuntersuchung blieb unauffällig, im Ultraschall war mastopathisches Drüsengewebe ohne einen abgrenzbar pathologischen Befund zu sehen. Die Krebsvorsorge am 17.8.2012 blieb ebenso ohne Tastbefund, aufgrund eines auffälligen Zervixabstrichs wurde jedoch eine Kontrolle für den 23.11.2012 vereinbart. Die Patientin stellte sich schon am 29.10.2012 wieder vor, da sie einen Knoten in der Brust spürte, den nun auch die Gynäkologin tastete. Der Ultraschall zeigte einen schwer abgrenzbaren, teils echoarmen, teils echoreichen Befund mit schmalem Randsaum ohne Schallschatten. Laut Gynäkologin sollte insoweit zunächst kurzfristig eine Kontrolle am ohnehin avisierten 23.11.2012 stattfinden. Diese Absprache bestritt die klagende Partei. Bei diesem Termin im November wurde auf jeden Fall aufgrund eines weiterhin auffälligen Tast- und Ultraschallbefunds eine Stanzbiopsie veranlasst, die ein gering differenziertes invasiv duktales Mammakarzinom ergab. Am 19.12.2012 wurde die rechte Brust nebst Achsellymphknoten entfernt; eine Re-OP zur Entfernung weiterer Tumoranteile folgte am 27.12.2012. Die Metastasierung schritt trotz Chemotherapie aber fort, die Patientin verstarb am 15.7.2015.

\section{So sah das Gericht den Fall}

Das Gericht wies die Klage ab (LG Frankfurt a.M., Urt. v. 27.4.2017, Az. 2-14 O 178/14). Laut Gutachter war am 16.7., 17.8. und 23.11.2012 kein Verstoß gegen fachärztliche Standards festzustellen. Im Juli 2012 hätten keine ausreichend diagnostischen Hinweise für ein Karzinom bestan- den, da die Patientin keine auffällige Anamnese hatte und Schmerzhaftigkeit, erst recht bei bestehender Regelblutung, untypisch für ein Karzinom sei, sondern eher für entzündliche Erkrankungen spreche. Nachvollziehbar - auch bei Rücküberlegungen aus späterer Tumorgröße und -biologie - sei, dass die Beklagte zwar knotige Verdichtungen, aber zunächst keinen auffälligen Herdbefund spürte. Ebenso zeigten auch die Ultraschallbilder nur eine Mastopathie und keine einzelne Herdstruktur. Daher habe auch noch keine Indikation für eine Mammografie oder gar Biopsie bestanden. Am 17.8.2012 sei der Verzicht auf einen erneuten Ultraschall bei rückläufigen Schmerzen, palpatorisch fehlendem Tastbefund und einer nur fünf Wochen zuvor unauffälligen Sonografie gleichfalls verständlich. Am 23.11.2012 seien schließlich alle gebotenen Maßnahmen veranlasst worden. Fehlerhaft sei allerdings die Reaktion am 29.10.2012 gewesen. Wegen des hier erstmals klar dokumentierten Herdbefunds bei Tast- und Ultraschalluntersuchung wäre sofort eine Mammografie zu veranlassen gewesen. Eine Verzögerung der Abklärung sei selbst bei Vorgabe eines Kontrollintervalls von vier Wochen nicht vertretbar, auch wenn Fehleinschätzung und ärztliches Handeln am 29.10. nicht als völlig unverständlich i. S. eines groben Behandlungsfehlers schienen. Eine Beweislastumkehr zulasten der Beklagten schied damit aus. Zudem hatten die streitigen Verzögerungen laut Gutachter schon aufgrund der Tumorbiologie keinen Einfluss auf den weiteren Verlauf. Dieser hätte sich selbst bei unterstellt früherer Diagnose mit an Sicherheit grenzender Wahrscheinlichkeit in gleicher Weise ergeben.

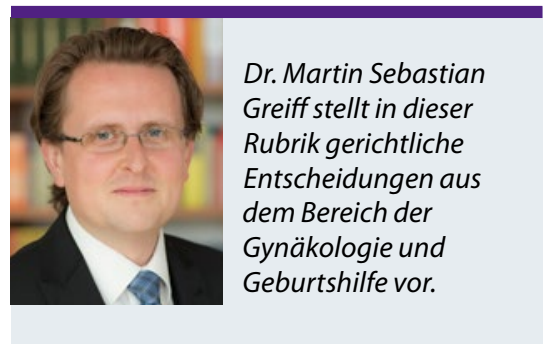

\section{Was bedeutet das Urteil für den klinischen Alltag?}

Die Entscheidung zeigt, wie kleinschrittig und detailliert jeder Arzt-Patienten-Kontakt in den Blick genommen wird. Vorliegend hatte die Ärztin am 29.10. den Befund immerhin selbst als kontrollbedürftig eingestuft, ihn in der Dokumentation mit BIRADS III notiert und wollte die Patientin kurzfristig wieder nach dem nächsten Pillenzyklus sehen. Die Empfehlung zur Vorstellung am 23.11. zur Kontrolle des fraglichen Brustbefunds wurde jedoch nicht als solche notiert, es fand sich nur die Notiz über die angesetzte Kontrolle des Zervixabstrichs. Daher nahm der Gutachter wohl zunächst an, dass die Kontrolle der Brust erst in drei bis sechs Monaten beabsichtigt gewesen wäre. Dieser Irrtum ließ sich in der Verhandlung korrigieren und für eine Haftung reichte die Sachlage mangels Nachweis haftungsbegründender Kausalität zwischen streitgegenständlichem Fehler und fraglichen Schäden nicht aus. Allein darauf sollte man sich aber nicht verlassen. Zum einen gibt es also an der Dokumentation wie immer Verbesserungsbedarf, zum anderen sollten verdächtige Befunde, so man sie schon selbst festhält, immer konsequent und sofort weiter abgeklärt werden.

Dr. Martin Sebastian Greiff, Mag. rer. publ. Rechtsanwalt und Fachanwalt für Medizinrecht, Partner der Kanzlei Ratzel Rechtsanwälte

Romanstraße 77, 80639 München www.ratzel-rechtsanwaelte.de 